\title{
“Guess Who ?” Large-Scale Data-Centric Study of the Adequacy of Browser Fingerprints for Web Authentication
}

\author{
Nampoina Andriamilanto, Tristan Allard, and Gaëtan Le Guelvouit
}

\begin{abstract}
Browser fingerprinting consists in collecting attributes from a web browser to build a browser fingerprint. In this work, we assess the adequacy of browser fingerprints as an authentication factor, on a dataset of 4,145,408 fingerprints composed of 216 attributes. It was collected throughout 6 months from a population of general browsers. We identify, formalize, and assess the properties for browser fingerprints to be usable and practical as an authentication factor. We notably evaluate their distinctiveness, their stability through time, their collection time, and their size in memory. We show that considering a large surface of 216 fingerprinting attributes leads to an unicity rate of $81 \%$ on a population of $1,989,365$ browsers. Moreover, browser fingerprints are known to evolve, but we observe that between consecutive fingerprints, more than $90 \%$ of the attributes remain unchanged after nearly 6 months. Fingerprints are also affordable. On average, they weigh a dozen of kilobytes, and are collected in a few seconds. We conclude that browser fingerprints are a promising additional web authentication factor.
\end{abstract}

\section{Introduction}

Web authentication widely relies on identifier-password pairs. Passwords are easy to use, but suffer from severe security flaws. Indeed, users use common passwords, paving the way to brute-force or guessing attacks [1]. They also reuse passwords

Nampoina Andriamilanto

Institute of Research and Technology $\mathrm{b}<>$ com, Cesson-Sévigné, France e-mail: nampoina . andriamilanto@b-com.com

Tristan Allard

Univ Rennes, CNRS, IRISA, Rennes, France e-mail: tristan.allardeirisa.fr

Gaëtan Le Guelvouit

Institute of Research and Technology $\mathrm{b}<>$ com, Cesson-Sévigné, France e-mail: gaetan .

leguelvouitab-com.com 
across websites [15] which increases the impact of attacks. Phishing attacks are also a major threat to passwords. Over the course of a year, Thomas et al. [13] achieved to retrieve 12.4 million credentials stolen by phishing kits. These flaws gave rise to multi-factor authentication [2], such that each additional authentication factor provides an additional security barrier. However, this usually comes at the cost of usability (i.e., users have to remember, possess, or do something).

In the meantime, browser fingerprinting gains attention. The seminal Panopticlick study [3] highlights the possibility to build a browser fingerprint by collecting attributes from a web browser. In addition to being widely used for web tracking purposes [4] (raising legal and ethical issues), browser fingerprints are used as an authentication factor in real-life. Browser fingerprints are indeed a good candidate as an authentication factor thanks to their distinctive power, their frictionless deployment (e.g., no additional software), and their usability (no secret to remember, no additional object to possess, and no supplementary action to carry out). As a result, companies like MicroFocus ${ }^{1}$ or SecureAuth ${ }^{2}$ include browser fingerprints within their authentication mechanisms (see Figure 1 for an example of such mechanism).

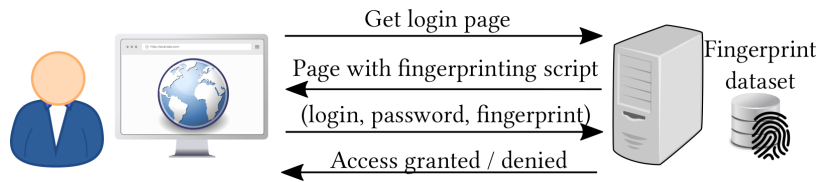

Fig. 1 Simplified web authentication mechanism relying on browser fingerprinting.

Related works. To the best of our knowledge, no large-scale study rigorously evaluates the adequacy of browser fingerprints as an authentication factor. Most works about their use for authentication concentrate on the design of an authentication mechanism $[14,10,6,11]$, and the empirical studies on browser fingerprints focus on their efficacy as a web tracking tool $[3,7,5]$. Such a mismatch between the understanding of browser fingerprints for authentication - currently poor - and their ongoing adoption in real-life is a serious harm to the security of web users. The lack of documentation from the existing tools (e.g., about the used attributes, about the distinctiveness of the resulting fingerprints, about their stability) only adds up to the current state of ignorance. All this whereas security-by-obscurity contradicts the most fundamental security principles.

Our contributions. We conduct the first large-scale data-centric empirical study of the fundamental properties of browser fingerprints when used as an additional authentication factor. We base our findings on an in-depth analysis of a real-life fingerprint dataset collected over 6 months, that contains 4,145,408 fingerprints composed of 216 attributes. We formalize, and assess on our dataset, the proper-

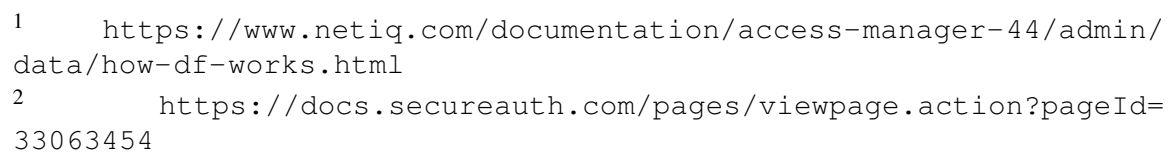


ties necessary for paving the way to elaborate browser fingerprinting authentication mechanisms. These properties are usually used to evaluate biometric characteristics [8]. We stress that we do not make any assumption on the inner working of the authentication mechanism, and consequently on the adversarial strategy. Our properties aim at characterizing the adequacy and the practicability of browser fingerprints, independently of their use within future authentication mechanisms. In particular, we measure the size of browser anonymity sets through time, and show that $81 \%$ of our fingerprints are unique. Moreover, we measure the proportion of identical attributes between two observations of the fingerprint of a browser, and show that $90 \%$ of the attributes remain unchanged after nearly 6 months. Finally, we measure the collection time and the size of fingerprints. We show that on average, they weigh a dozen of kilobytes, and are collected in a few seconds.

The rest of the paper is organized as follows. Section 2 presents and formalizes the properties evaluated in our analysis. Section 3 describes the analyzed dataset. Section 4 presents the experimental results. Finally, Section 5 synthesizes the results and concludes.

\section{Authentication Factor Properties}

The "Handbook of Fingerprint Recognition" [8] summarizes the properties that a biometric characteristic requires to be $u s a b l e^{3}$ as an authentication factor, and the additional properties required for a biometric authentication scheme to be practical. We make the link between the fingerprints used to recognize persons, and the ones used to recognize browsers. Therefore, we evaluate browser fingerprints according to these properties to assert their adequacy for web authentication. In this section, we list these properties, formalize how to measure some, and explain why the others are not addressed in this study.

The four properties needed for a biometric characteristic to be usable as an authentication factor are the following.

- Universality: the characteristic should be present in everyone.

- Distinctiveness: two distinct persons should have different characteristics.

- Permanence: the same person should have the same characteristic over time. We rather use the term stability.

- Collectibility: the characteristic should be collectible and measurable.

The three properties that a biometric authentication scheme requires to be practical are the following.

- Performance: the scheme should consume few resources, and be robust against environmental changes.

- Acceptability: the users should accept to use the scheme in their daily lives.

${ }^{3}$ Here, usable refers to the adequacy of the characteristic to be used for authentication, rather than the ease of use by the users. 
- Circumvention: it should be difficult for an attacker to deceive the scheme.

The properties that we study are the distinctiveness, the stability, and the performance. We consider that the universality and the collectibility are satisfied, as the HTTP headers that are automatically sent by browsers constitute a fingerprint. However, we stress that a loss of distinctiveness occurs when no JavaScript attribute is available. About the circumvention, we refer the reader to Laperdrix et al. [6] that analyzed the security of an authentication mechanism based on browser fingerprints. We let the evaluation of the acceptability as future works, but we stress that such mechanisms are already used in a rudimentary form ${ }^{4}$.

\subsection{Distinctiveness}

To satisfy the distinctiveness property, the browser fingerprints should distinguish two different browsers. The distinctiveness depends on the used attributes, and on the fingerprinted browser population. The two extreme cases are every browser sharing the same fingerprint, which makes them indistinguishable from each other, and no two browsers sharing the same fingerprint, making every browser distinguishable.

Our dataset entries are composed of a fingerprint, the source browser, and the time of collection in the form of a Unix timestamp in milliseconds. We denote $B$ the domain of the unique identifiers, and $T$ the timestamp domain. The fingerprint dataset is denoted $D$, and is formalized as:

$$
D=\{(f, b, t) \mid f \in F, b \in B, t \in T\}
$$

We use the size of the browser anonymity sets to quantify the distinctiveness, as the browsers that belong to the same anonymity set are indistinguishable. We denote $S(f, D)$ the function that returns the browsers that provided the fingerprint $f$ in the dataset $D$. It is formalized as:

$$
S(f, D)=\{b \in B \mid \forall(g, b, t) \in D, f=g\}
$$

We denote $A(\varepsilon, D)$ the function that provides the fingerprints that have an anonymity set of size $\varepsilon$ (i.e., that are shared by $\varepsilon$ browsers) in the dataset $D$. It is formalized as:

$$
A(\varepsilon, D)=\{f \in F \mid \operatorname{card}(S(f, D))=\varepsilon\}
$$

We measure the anonymity set sizes on the fingerprints currently in use by each browser, and not on their whole history. It is performed by simulating datasets composed of the last fingerprint seen for each browser at a given time. Let $E_{\tau}(D)$ be the simulated dataset originating from $D$ that represents the state of the fingerprints after $\tau$ days. With $t_{\tau}$ the last timestamp of this day, we have:

${ }^{4}$ https://support.google.com/accounts/answer/1144110 


$$
E_{\tau}(D)=\left\{\left(f_{i}, b_{j}, t_{k}\right) \in D \mid \forall\left(f_{p}, b_{q}, t_{r}\right) \in D, b_{j}=b_{q}, t_{r} \leq t_{k} \leq t_{\tau}\right\}
$$

\subsection{Stability}

Browser fingerprints have the particularity of evolving through time, due to changes in the web environment like a software update or a user configuration. We measure the stability by the mean similarity between two consecutive fingerprints observed for a browser, given the elapsed time between them. The two extreme cases are every browser holding the same fingerprint through its life, and the fingerprint of a browser changing completely between each observation.

We denote $C(\Delta, D)$ the function that provides the consecutive fingerprints of $D$ that are separated by a time lapse comprised in the $\Delta$ time range. It is formalized as:

$$
\begin{array}{r}
C(\Delta, D)=\left\{\left(f_{i}, f_{p}\right) \mid \forall\left(\left(f_{i}, b_{j}, t_{k}\right),\left(f_{p}, b_{q}, t_{r}\right)\right) \in D^{2},\right. \\
\left.b_{j}=b_{q}, t_{k}<t_{r},\left(t_{r}-t_{k}\right) \in \Delta\right\}
\end{array}
$$

We consider the Kronecker delta $\delta(x, y)$, being 1 if $x$ equals $y$, and 0 otherwise. We consider the set $\Omega$ of the $n$ used attributes. We denote $f[\omega]$ the value taken by the attribute $\omega$ for the fingerprint $f$. Let $\operatorname{sim}(f, g)$ be a simple similarity function between the fingerprints $f$ and $g$, which is formalized as:

$$
\operatorname{sim}(f, g)=\frac{1}{n} \sum_{\omega \in \Omega} \delta(f[\omega], g[\omega])
$$

We define the function meansim $(\Delta, D)$ that provides the mean similarity of the consecutive fingerprints, for a given time range $\Delta$ and a dataset $D$. It is formalized as:

$$
\text { meansim }(\Delta, D)=\frac{\sum_{(f, g) \in C(\Delta, D)} \operatorname{sim}(f, g)}{\operatorname{card}(C(\Delta, D))}
$$

\subsection{Performance}

We consider three aspects of the performance of browser fingerprints for authentication. They are the collection time of the fingerprints, their size in memory, and the loss of efficacy between the fingerprints of different device types.

We measure the collection time of our fingerprints composed of 200 JavaScript attributes, and ignore the HTTP headers that are transmitted passively. We measure the size of our fingerprints, having the canvas images [9] stored as sha256 hashes. We stress that compressing the fingerprint to a single hash is unpractical due to the evolution of fingerprints. Previous works showed that mobile and desktop devices present differences in the properties of their browser fingerprints $[12,7,5]$, notably 
that mobile browsers have less distinctive fingerprints. Following these findings, we assess that the properties of the fingerprints of mobile and desktop browsers are similar.

\section{Fingerprint Dataset}

To study the properties of browser fingerprints on a real-world browser population, we launched a fingerprint collection experiment. It was performed in collaboration with the authors of [5], and an industrial partner that controls one of the top 15 French websites according to Alexa ${ }^{5}$. The authors of [5] held the 17 attributes of their previous work [7] and focused on web tracking, whereas we held 216 attributes and focused on web authentication.

Table 1 Dataset comparison between Panopticlick, AmIUnique, Hiding in the Crowd, and this study. - denotes missing information, and * denotes deduced information. The attributes only comprise the original ones, and the fingerprints are counted after preprocessing.

\begin{tabular}{|c|c|c|c|c|}
\hline & PTC [3] & AIU [7] & HITC [5] & This study \\
\hline Collection period & 3 weeks & $3-4$ months* & 6 months & $\mathbf{6 ~ m o n t h s ~}$ \\
Number of attributes & 8 & 17 & 17 & $\mathbf{2 1 6}$ \\
Number of browsers & - & - & - & $\mathbf{1 , 9 8 9 , 3 6 5}$ \\
Number of fingerprints & 470,161 & 118,934 & $2,067,942$ & $\mathbf{4 , 1 4 5 , 4 0 8}$ \\
Number of distinct fingerprints & 409,296 & $142,023^{6}$ & - & $\mathbf{3 , 5 7 8 , 1 9 6}$ \\
Proportion of desktop fingerprints & - & $0.890^{*}$ & 0.879 & $\mathbf{0 . 8 0 5}$ \\
Proportion of mobile fingerprints & - & $0.110^{*}$ & 0.121 & $\mathbf{0 . 1 3 4}$ \\
Unicity of global fingerprints & 0.836 & 0.894 & 0.336 & $\mathbf{0 . 8 1 8}$ \\
Unicity of mobile fingerprints & - & 0.810 & 0.185 & $\mathbf{0 . 3 9 9}$ \\
Unicity of desktop fingerprints & - & 0.900 & 0.357 & $\mathbf{0 . 8 8 4}$ \\
\hline
\end{tabular}

\subsection{Fingerprint Collection}

We designed a fingerprinting probe that collects 200 JavaScript properties and 16 HTTP header fields. We integrated the probe to two general audience web pages of our industrial partner, which subjects are political news and weather forecast. The probe collected fingerprints from December 7, 2016, to June 7, 2017. Only the visitors that consented to cookies were fingerprinted, in compliance with the European directives 2002/58/CE and 2009/136/CE in effect at the time. To differentiate

5 https://www.alexa.com/topsites/countries/FR

${ }^{6}$ This number is provided in Figure 11 as the distinct fingerprints, but also corresponds to the raw fingerprints. Every fingerprint would be unique if the number of distinct and collected fingerprints are equal, hence we are not confident in this number, but it is the one provided by the authors. 
browsers, we assigned them a unique identifier (UID) as a 6-months cookie. Similarly to $[3,7]$, we coped with cookie deletion by storing a one-way hash of the IP address, computed by a secure cryptographic hash function.

Previous datasets were collected through dedicated websites, and are biased towards privacy-aware and technically-skilled persons $[3,7]$. Our population is more general audience oriented, but the website audience is mainly French-speaking users. This leads to a bias towards this population. The timezone is set to -1 for $98.48 \%$ of browsers, $98.59 \%$ of them have daylight saving time enabled, and $f r$ is present in $98.15 \%$ of the Accept-Language HTTP header.

\subsection{Dataset Filtering and Preprocessing}

Given the experimental aspect of fingerprints and the scale of our collection, the raw dataset contained erroneous or irrelevant samples. We remove 70,460 entries entries that have a wrong format (e.g., empty or truncated data), that are duplicated, or that come from a robot.

Cookies are an unreliable identification method, hence we perform a resynchronization similar to [3]. We consider the entries that have the same (fingerprint, IP address hash) pair to come from the same browser, and assign them the same UID. Similarly to [3], we do not synchronize the interleaved UIDs, that are the pairs having the UID values $b_{1}, b_{2}$, then $b_{1}$ again. We replace 181,676 UIDs with 116,708 replacement UIDs using this method.

To avoid counting multiple entries of identical fingerprints coming from the same browser, the usual way is to ignore them during collection [3, 7]. Our probe collects the fingerprint on each visit, and to stay consistent with common methodologies we deduplicate the fingerprints afterward. For each browser, we hold the first entry that has a given fingerprint, and ignore the consecutive entries if they have this fingerprint. For example, if a browser $b$ has the entries $\left\{\left(f_{1}, b, t_{1}\right),\left(f_{2}, b, t_{2}\right),\left(f_{2}, b, t_{3}\right),\left(f_{1}, b, t_{4}\right)\right\}$, we hold $\left\{\left(f_{1}, b, t_{1}\right),\left(f_{2}, b, t_{2}\right),\left(f_{1}, b, t_{4}\right)\right\}$. The deduplication constitutes the biggest cut in our dataset, with 2,420,217 entries filtered out.

We extract 46 additional attributes from 9 original attributes, which are of two types. The first type consists in extracted attributes composed of parts of their original attribute, like the screen resolution that is split into the values of the width and the height. The second type consists of information sourced from an original attribute, like the number of plugins extracted from the list of plugins.

\subsection{Work Dataset}

The work dataset obtained after the preprocessing step contains 5,714,738 entries (comprising identical fingerprints for a given browser if they are interleaved), 
with 4,145,408 fingerprints (no identical fingerprint counted for the same browser), and composed of 3,578,196 distinct fingerprints. The fingerprints are composed of 216 original attributes and 46 extracted ones, for a total of 262 attributes. They come from 1,989, 365 browsers, $27.53 \%$ of which have multiple fingerprints. Table 1 presents a comparison between the dataset of Panopticlick [3], AmIUnique [7], Hiding in the Crowd [5], and this study.

\section{Empirical Evaluation of Browser Fingerprints Properties}

In this section, we evaluate the browser fingerprints of our dataset according to the properties presented in Section 2. We show that our fingerprints offer a satisfying distinctiveness, as $81 \%$ of them are only shared by one browser. Moreover, our fingerprints are stable, as more than $90 \%$ of the attributes are expected to stay identical between two observations, even if they are separated by nearly 6 months. Our fingerprints of mobile browsers are less distinctive than the fingerprints of desktop browsers, with a unicity rate of $42 \%$ against $84 \%$. Finally, our fingerprints do not hinder the performance as, on average, they weigh a dozen of kilobytes and take a few seconds to collect.

\subsection{Distinctiveness}

We call unicity rate the proportion of the fingerprints that are shared by a single browser. Our fingerprints offer a satisfying distinctiveness, as they have a stable unicity rate of approximately $81 \%$ on the long run, and more than $94 \%$ of the fingerprints are shared by 8 browsers or less. However, the fingerprints of the mobile browsers are more uniform than the fingerprints of the desktop browsers, with a unicity rate of approximately $42 \%$ against $84 \%$, on the long run.

Figure 2 presents the size of the anonymity sets (AS) alongside the frequency of browser arrival for the daily-partitioned datasets. New browsers are encountered continually, but starting from the 60th day, the arrival frequency stabilizes around 5,000 new browsers per day. Before this stabilization, we have a variable arrival frequency with some major spikes that seem to correspond to events that happened in France. For example, the spike on the 38th day corresponds to a live political debate on TV, and the spike on the 43rd day correlates with the announcement of a cold snap.

Figure 3 presents the unicity rate through the partitioned datasets for the overall, the mobile, and the desktop groups. The unicity rate is stable for the desktop browsers, with a slight increase of 1.04 points from the 60th day to the 183th, from $84.99 \%$ to $86.03 \%$. The unicity rate of the mobile browsers is lower, and it has a little decrease of 0.29 points on the same period, from $42.42 \%$ to $42.13 \%$. 


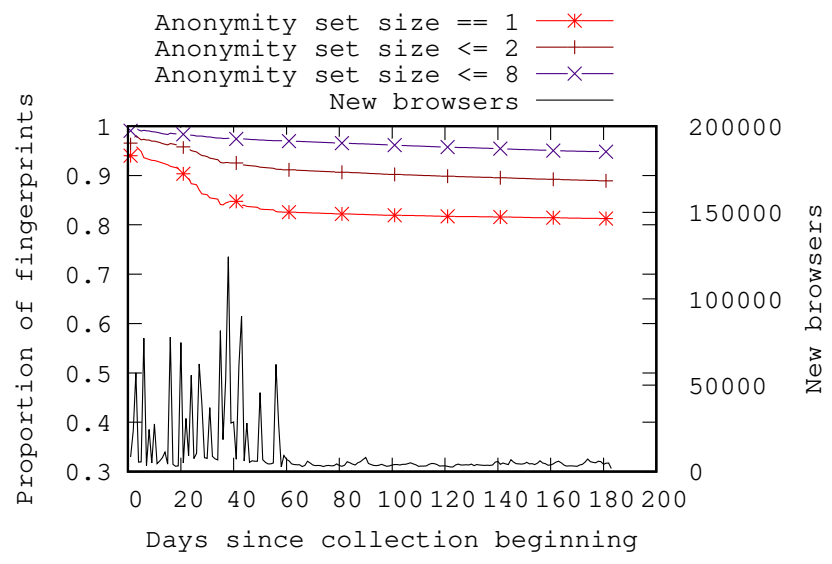

Fig. 2 Anonymity set sizes and frequency of browser arrivals through the partitioned datasets obtained after each day.

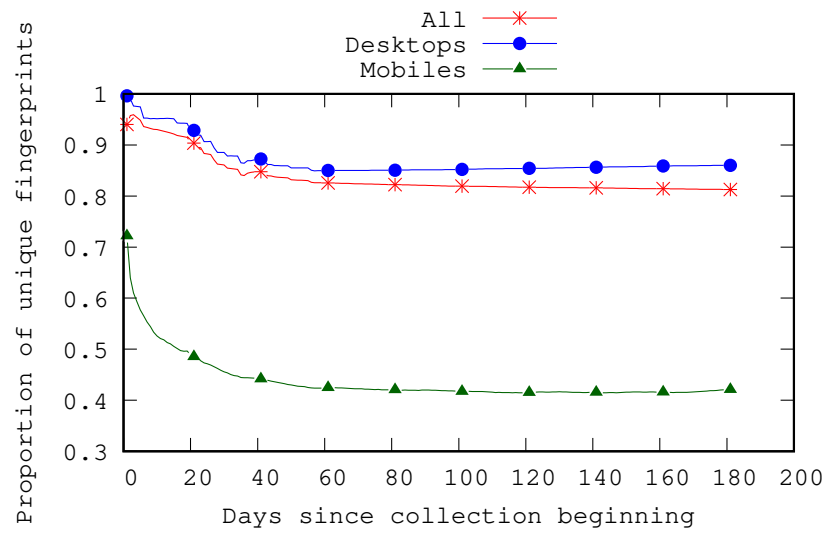

Fig. 3 Proportion of unique fingerprints for the overall, the mobile, and the desktop groups, through the partitioned datasets obtained after each day.

\subsection{Stability}

Our fingerprints are stable, as a browser is expected to have at least $90 \%$ of its attributes unchanged, even after 170 days. The fingerprints of the mobile browsers are generally more stable than the fingerprints of the desktop browsers.

Figure 4 displays the mean similarity between the consecutive fingerprints in function of the time difference. The ranges $\Delta$ are expressed in days, so that day $d$ on the $\mathrm{x}$-axis represents the fingerprints separated by $\Delta=[d ; d+1[$ days. We ignore the comparisons of the time ranges that have less than 10 pairs, or that have a time difference higher than the limit of our experiment (182 days). These outliers account for less than $0.03 \%$ of each group. The results are obtained by comparing 
$3,725,373$ pairs for the overall group, 2,912,860 pairs for the desktop group, and 594,591 pairs for the mobile group.

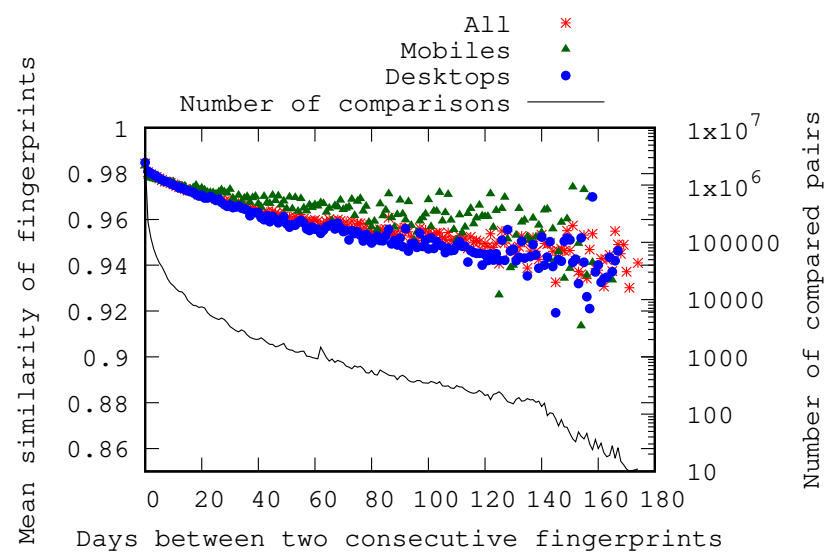

Fig. 4 Mean similarity between the consecutive fingerprints in function of the time difference, with the number of compared pairs, for the overall, the mobile, and the desktop groups.

\subsection{Performance}

\subsubsection{Time Resource Consumption}

Our probe takes several seconds to collect the attributes that compose our fingerprints. The median collection time of our fingerprints is of 2.92 seconds. Mobile browsers take more time to provide the collected attributes, with a median collection time of 4.44 seconds, against 2.64 seconds for the desktop browsers. This is less than the median loading time of web pages ${ }^{7}$, which is of 6.6 seconds for the desktop browsers, and of 19.6 seconds for the mobile browsers, at the date of March 1, 2020.

Figure 5 displays the cumulative distribution of the collection time of our fingerprints. We measure the collection time by the time difference between the starting of the script and the fingerprint sending. Some values take from several hours to days. They can come from a web page that is put in background or accessed after a long time. We limit our population to the fingerprints that take less than 30 seconds to collect, and consider the higher values as outliers. The outliers account for less than $1 \%$ of each group.

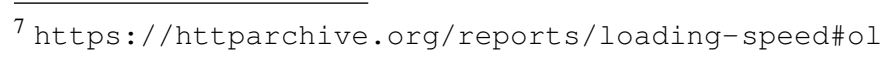




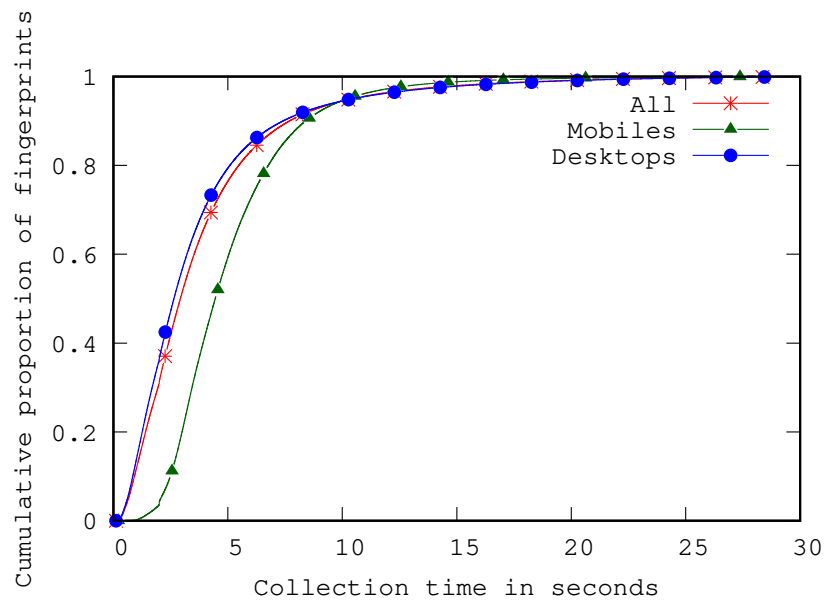

Fig. 5 Cumulative distribution of the collection time of the fingerprints in seconds, for the overall, the mobile, and the desktop groups.

\subsubsection{Memory Resource Consumption}

Our script consumes a dozen of kilobytes per fingerprint, a size that is easily handled by the current storage and bandwidth capacities. Half of our fingerprints weigh less than 7,550 bytes, and $99 \%$ less than 14 kilobytes. The fingerprints of the desktop browsers are heavier, with $95 \%$ of the fingerprints weighing less than 12,082 bytes, against 8,020 bytes for the fingerprints of mobile browsers. This is due to heavy attributes being lighter on mobile browsers, like the list of plugins that is usually empty.

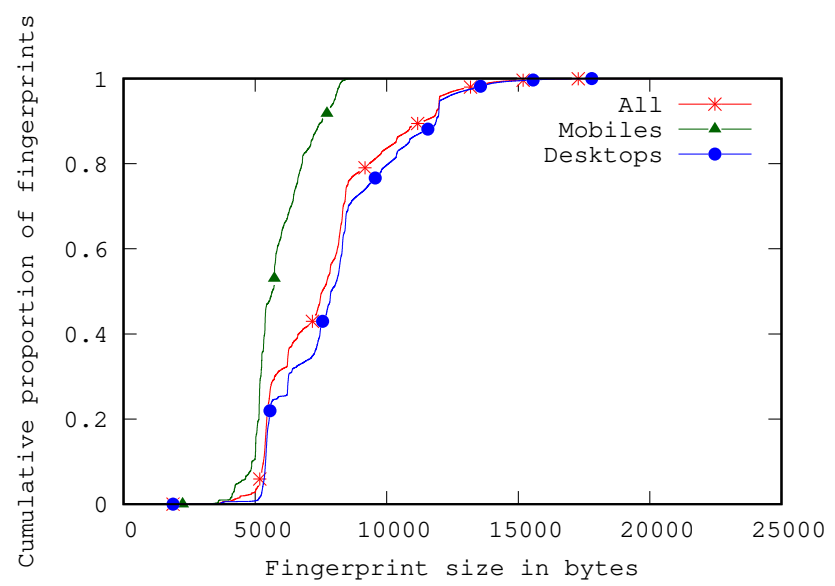

Fig. 6 Cumulative distribution of the fingerprint size in bytes, for the overall, the mobile, and the desktop groups. 
Figure 6 displays the cumulative distribution of the size of our fingerprints in bytes. The average fingerprint size is $\mu=7,692$ bytes, and the standard deviation is $\sigma=2,294$. We remove 1 fingerprint from a desktop browser that is considered an outlier because of its size being greater than $\mu+15 \cdot \sigma$.

\section{Synthesis of Results and Conclusion}

In this study, we evaluate the properties offered by browser fingerprints as an additional web authentication factor, through the analysis of a large-scale real-life fingerprint dataset. We show that browser fingerprints offer a satisfying distinctiveness, as $81 \%$ of our fingerprints are only shared by one browser. Moreover, fingerprints are stable. At least $90 \%$ of our attributes are expected to stay identical between two observations of the fingerprint of a browser, even if the observations are separated by nearly 6 months. We validate that fingerprints offer a high performance, as they only weigh a dozen of kilobytes, and take a few seconds to collect. We conclude that browser fingerprints provide satisfying properties for an additional web authentication factor, and can strengthen password-based systems without a major loss of usability.

Acknowledgements We want to thank Benoît Baudry and David Gross-Amblard for their valuable comments, together with Alexandre Garel for his work on the experiment. This is a postprint of a contribution published in Innovative Mobile and Internet Services in Ubiquitous Computing, edited by Leonard Barolli, Aneta Poniszewska-Maranda, and Hyunhee Park, and published by Springer International Publishing. The final authenticated version is available online at: https://doi.org/10.1007/978-3-030-50399-4_16.

\section{References}

1. Bonneau, J.: The science of guessing: analyzing an anonymized corpus of 70 million passwords. In: S\&P (2012)

2. Bonneau, J., Herley, C., van Oorschot, P.C., Stajano, F.: Passwords and the Evolution of Imperfect Authentication. Communications of the ACM (2015)

3. Eckersley, P.: How unique is your web browser? In: PETS (2010)

4. Englehardt, S., Narayanan, A.: Online Tracking: A 1-million-site Measurement and Analysis. In: CCS (2016)

5. Gómez-Boix, A., Laperdrix, P., Baudry, B.: Hiding in the Crowd: an Analysis of the Effectiveness of Browser Fingerprinting at Large Scale. In: TheWebConf (2018)

6. Laperdrix, P., Avoine, G., Baudry, B., Nikiforakis, N.: Morellian analysis for browsers: Making web authentication stronger with canvas fingerprinting. In: DIMVA (2019)

7. Laperdrix, P., Rudametkin, W., Baudry, B.: Beauty and the Beast: Diverting modern web browsers to build unique browser fingerprints. In: S\&P (2016)

8. Maltoni, D., Maio, D., Jain, A.K., Prabhakar, S.: Handbook of Fingerprint Recognition, pp. 8-11. Springer-Verlag (2003)

9. Mowery, K., Shacham, H.: Pixel perfect: Fingerprinting canvas in html5. In: W2SP (2012) 
10. Preuveneers, D., Joosen, W.: SmartAuth: Dynamic Context Fingerprinting for Continuous User Authentication. In: SAC (2015)

11. Rochet, F., Efthymiadis, K., Koeune, F., Pereira, O.: SWAT: Seamless web authentication technology. In: TheWebConf (2019)

12. Spooren, J., Preuveneers, D., Joosen, W.: Mobile Device Fingerprinting Considered Harmful for Risk-based Authentication. In: EuroSec (2015)

13. Thomas, K., Li, F., Zand, A., Barrett, J., Ranieri, J., Invernizzi, L., Markov, Y., Comanescu, O., Eranti, V., Moscicki, A., et al.: Data breaches, phishing, or malware?: Understanding the risks of stolen credentials. In: CCS (2017)

14. Unger, T., Mulazzani, M., Frühwirt, D., Huber, M., Schrittwieser, S., Weippl, E.: SHPF: Enhancing HTTP(S) Session Security with Browser Fingerprinting. In: ARES (2013)

15. Wang, C., Jan, S.T., Hu, H., Bossart, D., Wang, G.: The next domino to fall: Empirical analysis of user passwords across online services. In: CODASPY (2018) 